\author{
A C TA U N I E R S TAT IS L O D Z I E N S IS \\ FOLIA GERMANICA 11, 2015
}

http://dx.doi.org/10.18778/1427-9665.11.04

Katarzyna Sikorska-Bujnowicz*

\title{
FRAUEN-MÄNNER-WITZ FRÜHER UND HEUTE. EINIGE BEMERKUNGEN ZUM DEUTSCHEN KALAUER
}

\section{Zielsetzung}

In diesem Artikel wird versucht, die Frage nach der Zuordnung eines humoristischen Textes zur Gruppe der Kalauer im Deutschen zu beantworten. Nicht ohne Bedeutung bleibt dabei die Antwort auf die Frage nach der Definition eines Kalauers und danach, welche Rolle den von den Autoren absichtlich eingesetzten Verstößen gegen alle verbindlichen sprachlichen und sozialen Normen zugeschrieben steht, die als Mittel des Humoristischen verstanden werden.

Das Analysematerial bilden hier die ausgewählten Texte von Loriot ${ }^{1}$ (aus den 70er Jahren des 20. Jahrhunderts), Sketchtexte aus dem deutschen Programm „Was guckst du?" ${ }^{2}$ und Witze von heute, die auf den deutschen Internetseiten zu finden sind. In diesem Beitrag werden die repräsentativsten Beispiele angeführt und besprochen. Alle Zitate stehen in der nicht korrigierten Originalform.

\section{Zum Witzbegriff}

In der Humorforschung wird in erster Linie darauf gezeigt, welche Merkmale ein Text aufweisen soll, um als ein humoristischer eingestuft zu werden. Die Untersuchungen in diesem Bereich hängen auch mit den zahlreichen Definitionen vom

* Dr. Katarzyna Sikorska-Bujnowicz, Universität Łódź, Philologische Fakultät, Lehrstuhl für Deutsche und Angewandte Sprachwissenschaft, 90-236 Łódź, Pomorska 171/173.

${ }^{1}$ Loriot ist das Pseudonym von Bernhard-Viktor Christoph-Carl von Bülow (kurz Vicco von Bülow, 1923-2011). Man nennt ihn einen der besten deutschen Humoristen aller Zeiten. Sein ganzes Leben lang war er mit der Unterhaltungskunst verbunden, wurde zuerst vor allem dank seiner Cartoons bekannt, die in den 50er Jahren in der Zeitung „Die Straße“ und im „Stern“ erschienen sind. Die bekanntesten Sketchtexte sind in den 70er Jahren im Radio Bremen präsentiert worden und sind inzwischen auch auf DVD erhältlich.

${ }^{2}$ Das Programm „Was guckst du?“ ist ein multikulturelles Comedy-Programm, das von einem türkisch-arabischen, in Deutschland geborenen Komiker - Kaya Yanar - ins deutsche Fernsehen gebracht und dort moderiert wurde. Für seine Sendung bekam er zahlreiche Preise, u.a. den Deutschen Fernsehpreis 2001 in der Kategorie „Beste Comedy“. 
Lächeln und dem Lachen zusammen, wobei vor allem auf Freud (1905/1985) und auf Bergson (1900/1977) als Gründer der Humorforschung hingewiesen werden soll, bei denen an erster Stelle die bestehenden Zusammenhänge zwischen dem Lächeln/Lachen und der menschlichen Psyche genannt werden. Der Mensch als Element einer sozialen Gruppe zeichnet sich dadurch aus, dass nur für ihn das Lachen charakteristisch ist, dem Bergson (1900/1977) die sog. soziale und erzieherische Funktion zuschreibt. Wie werden dann Kalauer platziert, die als schlechte Witze zu verstehen sind? Neben den genannten Funktionen soll ein Witz nämlich noch eine informative besitzen (Plessner 1941/1982), weil als Reaktion auf etwas, was sprachlich nicht zu beantworten ist, eben das Lachen erwartet wird.

In der Literatur zur Humorforschung lesen wir des Weiteren u.a. bei Bremmer und Roodenburg (1999), dass der Humor als etwas Neutrales zu verstehen ist. Wie kann aber etwas Neutrales als ein schlechter Witz klassifiziert werden? Die beiden Autoren weisen nämlich darauf hin, dass ein Witz eine gezielte und nicht zufällig formulierte Botschaft ist, die das Lächeln oder das Lachen hervorrufen soll, was auf den ontologischen Charakter dieser Humortheorie zurückgeht.

Wenn man über die Klassifikation der Witze spricht, darf man nicht vergessen, welche Rolle dem Ethnowitz zugeschrieben wird, mit dem Stereotype verschiedener Art zusammenhängen, die als Wiederspiegelung der von Generationen vererbten Vorstellungen und Ideen sind (Sikorska-Bujnowicz 2013, S. 18). So wird der Humor auch von Plessner definiert, dem er einen nationalen Charakter zuschreibt. Das alles trägt demzufolge dazu bei, dass ein und derselbe, als ein humoristischer gedachter Text verschieden interpretiert werden kann, wobei die Sprache als Hauptmittel gemeint wird, deren Kenntnis ein notwendiges Element der scherzhaften Kommunikation ist. ${ }^{3}$ Für die Rezipienten ist es wichtig, über die Kenntnisse der jeweiligen Sprache zu verfügen, was mit allen Bedeutungsvarianten, übertragenen Bedeutungen und den kleinsten Nuancen zusammenhängt. Erst dieses Wissen gibt dem Leser bzw. Hörer die Möglichkeit, einen Text als einen humoristischen wie erwartet zu interpretieren und zum Schluss natürlich über das Gelesene bzw. Gehörte zu lachen. Das bestätigen die Ergebnisse der für die Zwecke dieses Beitrags durchgeführten Analyse, aus der eindeutig hervorgeht, dass neben den sprachlichen Kenntnissen auch das Vorwissen des Rezipienten verlangt wird. Nur auf diese Weise können die von den Autoren versteckten Botschaften erkannt, richtig verstanden und zum Schluss interpretiert werden.

Auf der kommunikativen Ebene versteht man den Humor als Befriedigung der Aggression, des Triebs zum Tabubruch und des Spieltriebs, was auf Freud zurückgeht. In diesem Bereich werden die Zusammenhänge zwischen Produktion und Rezeption der Witze genannt.

${ }^{3}$ Nicht weniger wichtig ist natürlich auch die Übersetzbarkeit der Witze, mit der sich u.a. Dimova (2000) beschäftigt. 


\section{Witz als Element der Sprache}

Wenn man den Witz als Element der Sprache zu definieren versucht, soll man in erster Linie darauf hinweisen, welche weiteren Fragen beantwortet werden müssen, wenn man einen Text als einen humoristischen einzustufen beabsichtigt. An dieser Stelle sollten das Thema, die Zielscheibe und die Pointe genannt werden (Raskin 1985, Raskin 2008, Titze 2009a, Titze 2009b), die als Bestandteile jedes witzigen Textes zu verstehen sind. Die sprachliche Analyse der Witze hängt mit dem Begriff des Skripts zusammen, was bei Raskin (1985) die Grundeinheit der Semantischen Skripttheorie ist. Darunter wird ein semantisches Feld definiert, in dem alle Informationen stehen, die mit den im jeweiligen Witz gebrauchten lexikalischen Einheiten verbunden sind und deren Aufgabe ist, dem Rezipienten das Verstehen der versteckten Inhalte zu erleichtern, was vor allem mit den von ihnen hervorzurufenden Assoziationen zusammenhängt. Die von Raskin in der erwähnten Theorie genannte Ambiguität steht an der Spitze aller sprachlichen Mittel des Humoristischen, weil sie das Abrufen zahlreicher Assoziationen bewirkt und somit das richtige Entschlüsseln der versteckten Inhalte und die damit zusammenhängende Interpretation beeinflusst. Das alles verlangt aber von dem Rezipienten das konkrete Vorwissen und die Sprachkenntnisse.

Wie ist es im Falle eines Kalauers? Achtet der Empfänger darauf, was sprachlich zum Ausdruck gebracht wird oder orientiert er sich eher nach dem Thema, der Zielscheibe und dem Motiv? Solche Fragen werden natürlich verschieden beantwortet, was auf den jeweiligen Rezipienten zurückgeht. Wer über das geringe Wissen über die Sprache verfügt und sich nur danach richtet, ob er nach dem gehörten Witz zu lachen anfängt oder nicht, dem fällt es viel leichter, humoristische Texte als gut bzw. schlecht einzustufen, was in diesem Falle einerseits auf seinen Sinn für Humor zurückgeht bzw. dessen Fehlen oder darauf, was er unter dem Begriff des guten bzw. schlechten Geschmacks versteht, der bei der Interpretation der Witze nicht außer Acht gelassen werden soll.

Die Allgemeine Theorie des Verbalen Humors (General Theory of Verbal Humour) von Attardo und Raskin (1991) gilt als Erweiterung der bereits genannten Skripttheorie. Die Autoren weisen hier auf weitere, die die in der Skripttheorie genannte semantische Ebene ergänzenden Elemente. Es sind Regeln, die das Umschalten von Skript zum Skript bestimmen und die Bestandteile des Witzes, d.h. die Zielscheibe, das Thema, die Pointe und die Gattung.

Die Humorforscher zeigen auch darauf, welche sprachlichen Mittel zum Erzielen des Humoristischen dienen und welche Rolle die Sprache als Kommunikationsmittel hat.

In der deutschen Humorforschung kann man sich vor allem auf Helga Kotthoff berufen, die sich mit verschiedenen Problemen im Rahmen des Humoristischen auseinandersetzt, d.h. sie schreibt u.a. über den konversationellen Humor 
(1994, 1996, 1997a, 1997b, 2003a, 2007), über die Intertextualität (2000, 2002b), über Humor und Medien (2002a, 2004a, 2006), über den Ethnowitz (2009) und auch über die Gendersprachen in Bezug auf die Humoraspekte (1986, 1988, 1992, 1998, 2000, 2002, 2006).

Auf dem Gebiet der polnischen Humorforschungen war Danuta Buttler (1968/2001) die erste, die versucht hat, den polnischen Sprachwitz zu untersuchen. Als Untersuchungsmaterial wählte Buttler die Zitate aus Büchern und Zeitschriften, wobei sie sich auf einen Zeitraum von sechzig Jahren bezog (1900 bis Anfang der 60er Jahre). In der dritten Ausgabe ihres Buches aus dem Jahre 2001 stößt man auf eine interessante Aussage von Chlebda, der darauf hinweist, dass die Sprache des 21. Jahrhunderts schon eine andere ist, was in Bezug auf den Humor nicht ohne Bedeutung bleibt. Was bei Buttler früher mit dem Vermerk „obszön“, „vulgär“" versehen wurde, gilt heute manchmal sogar als neutral, was davon zeugt, dass sich einerseits die Tabugrenzen verschoben haben und andererseits auch die sozialen Normen anders sind als die, die in dem genannten Zeitraum galten. Man kann an dieser Stelle das Zitat von Chlebda anführen:

(...) zwłaszcza minione dziesięciolecie przesunęło granicę cenzuralności w rejony, których Profesor Buttlerowa nie mogłaby sobie wyobrazić, a dowcipy znacznie gorsze przynoszą dziś dzieci z przedszkola" (Buttler 2001, Einleitung S.V).

Das ist ein weiterer Beweis dafür, dass die humoristischen Texte auch diachron untersucht werden können, damit gezeigt werden kann, wie sich die Sprache als Mittel des Humoristischen im Laufe der Zeit entwickelt hat.

Wie es in der Einleitung geschrieben steht, ist dieser Beitrag ein Versuch, den deutschen Kalauer früher und heute zu besprechen, d.h. zu zeigen, was innerhalb der Sprache als Inventar der Mittel des Humoristischen konstant geblieben ist und was sich eventuell verändert hat.

\section{Zum Frauen-Männer-Witz}

Wenn man Witze als eine sehr große Gruppe von Texten analysiert, fällt es auf, dass eben die Frauen-Männer-Witze an der zweiten Stelle, nach den Berufe-Witzen, stehen. In erster Linie ist hier die Frau als Thema und zugleich Zielscheibe aufzufassen, wobei die Motive unterschiedlich sein können, z.B. Liebe zu den Schuhen

(1) Aschenputtel MUSS ein Märchen sein. Keine Frau dieser Welt würde in der Realität einen Schuh zurücklassen, solange sie noch zwei Beine hat.

oder der Wunsch, immer das letzte Wort zu haben

(2) Sagt eine Frau zu ihrem Mann:,,Sag mal, findest Du auch, dass ich so furchtbar dominant bin?” Da fällt der Mann auf die Knie und antwortet: „Aber nein, meine Herrin!” 
Mit im Spiel sind auch die Männerfiguren, denen in einem Witz manchmal auch die Hauptrolle zugeschrieben wird, wobei doch der Rezipient zwischen den Zeilen lesen kann und den Hauptgedanken entschlüsselt, d.h. die Frau steht immer an der Spitze. Die Männer kritisieren ihre Frauen und machen sie sogar lächerlich, z.B.

(3) Forscher haben herausgefunden, dass sich in Bier massenhaft weibliche Hormone befinden. Das hab ich mir schon immer gedacht! Jedes Mal, wenn ich vier oder fünf Maß trinke, rede ich nur noch dummes Zeug daher und kann nicht mehr ordentlich Auto fahren.

(4) „Führt deine Freundin Selbstgespräche?” - „Ja, aber sie weiß nichts davon. Sie denkt immer, ich höre zu".

(5) ,Warum bist du so gut gelaunt?“ - „Gestern Morgen sagte mein Chef zu mir, dass ich befördert werde und mein Gehalt verdoppelt wird. Mittags kontrollierte ich die Lottozahlen und stellte fest, dass ich 6 Richtige plus Superzahl habe. Als ich abends nach Hause kam, saß meine Frau auf gepackten Koffern und verließ mich. Wenn es einmal läuft, dann läuft es!“‘

(6) Ein Mann sitzt an der Bar und weint. Der Kellner fragt ihn: „Haben Sie Kummer? “ „Meine Frau hat mir gesagt, sie würde einen Monat kein Wort mehr mit mir reden.” „Wie schrecklich“. ,Ja, heute ist der Monat um“.

In einem Witz, der zur Gruppe der Frauen-Männer-Witze gehört wird auch mit solchem Motiv gespielt, dass der Mann als Witzfigur sogar vom Tod seiner Frau träumt, z.B.

(7) „Meine Frau ist ein Engel!” - „Hast du ein Glück! Meine lebt noch!“

Auch wenn es nicht um seine verhasste Frau geht, sondern um ein Haustier, assoziiert der Mann alles mit ihr:

(8) Der Tierarzt ruft bei Herrn Meier an: „Ihre Frau ist mit Ihrer Katze da und bat mich, sie einzuschläfern. Ist das in Ordnung?“ „Klar, und die Katze können Sie raussetzen, sie kennt den Heimweg“".

Hier verstößt man nicht selten gegen gesellschaftliche Normen, wenn sogar in dieses Hass-Spiel zwischen seinen Eltern ihr Kind verwickelt wird, z.B.

(9) Sohn beim Kreuzworträtsel: „Papa, kennst du ein Küchengerät mit vier Buchstaben?“ Vater zum Sohn: „Jepp! Frau!“‘

In den Frauen-Männer-Witzen wird nicht selten das Verhalten der Frauen dem der Männer gegenübergestellt, z.B.

(10) Worüber reden drei Männer auf einer einsamen Insel? Frauen, Fußball und Autos. Worüber reden drei Frauen auf einer einsamen Insel? Zwei tun sich zusammen und reden über die dritte. 
Unter den Frauen-Witzen kann eine besonders große Gruppe unterschieden werden, in der eine Blondine gleichzeitig als Zielscheibe und Thema funktioniert. Hier wird vor allem eine Frage-Antwort-Struktur gebraucht, in der am meisten W-Fragen auftauchen, z.B.

(11) Was machen zwei Blondinen vor dem Kino? - Sie warten auf die 16 anderen, weil der Film erst ab 18 ist.

(12) Was macht eine Blondine wenn es blitzt? Sie steigt auf's Dach und macht Posen weil sie denkt, dass sie fotografiert wird.

(13) Warum läuft eine Blondine beim Duschen ständig hin und her? - Weil auf dem Duschgel steht: „Wash and go”.

(14) Zwei Blondinen unterhalten sich, sagt die eine: „Du ich habe einen Schwangerschaftstest gemacht!” Darauf die andere: „Und, waren die Fragen schwer?”

(15) Sind zwei Blondinen in der Dusche. Sagt die eine zur anderen: „Kannst du mir dein Shampoo geben?” Antwortet die andere: „Du hast da doch deins!” Daraufhin sagt die andere wieder. „Nein, hier steht für trockenes Haar, aber meine sind doch nass...”

Nicht selten erscheint eine blonde Frau in einem makabren Witz, z.B.

(16) Sagt die eine Blondine zur anderen: „Du, der Wilhelm wird nicht jetzt am Freitag beerdigt, sondern erst am Freitag in acht Tagen.“ Antwortet die andere: „Ah, geht's ihm wieder besser?“

In den Witzen dieser Art steht vor allem das fehlende Wissen der Witzfiguren im Vordergrund. Wie können solche Witze bewertet werden - sind sie gut oder vielleicht doch schlecht? Wie im Falle jedes humoristischen Textes wird es dem Hörer bzw. Leser überlassen, was auf die Subjektivität in Bezug auf den Humor zurückgeht. Wichtig sind demzufolge Fragen nach der Definition eines Kalauers.

\section{Zum Kalauer}

In DUDEN stoßen wir auf folgende Definition des Kalauers:

Ka|lauler, der; -s, - [unter Anlehnung an frz. calembour $(\mathrm{g})=$ Wortspiel geb. nach dem Namen der Stadt Calau bei Cottbus]: nicht sehr geistreicher, meist auf einem Wortspiel beruhender Witz: einen K. erzählen; Gelang dem einen immerhin noch hin und wieder ein Scherzchen, so wollten dem anderen nicht einmal die K. glücken (Zeit 19.9. 75, 42).

Duden Universalwörterbuch CD-ROM C 2000 Dudenverlag

Aus der zitierten Definition geht hervor, dass mindestens zwei Aspekte verschiedener Art genannt werden - ein Wortspiel auf der sprachlichen Ebene und das Merkmal ,nicht sehr geistreich“ auf der Ebene der Wahrnehmung und des damit zusammenhängenden Geschmacks. 
Interessant ist dabei die Geschichte des Wortes Kalauer selbst, das im Deutschen seit den 50er Jahren des 19. Jahrhunderts als Bezeichnung eines schlechten, billigen Witzes zu finden ist. ${ }^{4}$ Der Kalauer hängt mit dem Namen einer brandenburgischen Kleinstadt Kalau zusammen, was im DUDEN Herkunftswörterbuch folgendermaßen definiert wird:

Kalauer fauler [Wort]witz: Die seit 1858 bezeugte Bezeichnung ist wohl eine Umformung von frz. calembour Wortspiel nach dem Namen der niederlausitzischen Stadt Kalau. Abl.: kalauern Kalauer machen (20. Jh.).

(C) Dudenverlag, Sat_Wolf, Bayern

Die Geschichte geht auf das 19. Jahrhundert zurück, als im Jahre 1833120 Schuster lebten und arbeiteten. Es wird erzählt, dass das Erzählen der Witze bei langer Arbeit geholfen haben soll. Der Satiriker Ernst Dohm hielt sich oft in Calau (früher noch mit K geschrieben - Kalau) auf und notierte diese witzigen Geschichten. In Berlin veröffentlichte er dann diese Witze in Form einer wöchentlichen Zeitschrift „Kladderadatsch“.

Dabei gibt es noch eine weitere Erklärung dieses Begriffes, die von den Sprachpuristen formuliert wird, nämlich das Zurückführen des Wortes Kalau auf das französiche calembour, das die Bedeutung, Wortspiel' oder, fauler Witz' hat.

An dieser Stelle sollten ein paar Witze genannt werden, die von den Einwohnern von Calau ${ }^{5}$ erzählt werden:

(17) „Warum können die Beamten nicht versetzt werden? Sie werden umgebettet!“

(18) „Wo wohnt die Katze? Im Miezhaus!“

(19) „Warum haben in Calau so viele Häuser und Schuppen Flachdächer? Damit die Hypotheken besser aufgelegt werden können!“

(20) „Warum hat Korbmacher Krause keine Kopfhaare mehr auf dem Kopf? Weil die Afrikaner Krauses Haar haben!“

An den genannten Beispielen kann erkannt werden, wie verschieden ein witziger Text sein kann. Man geht einerseits von dem Wortspiel aus, z.B. (17) versetzen vs. umbetten, (19) flache Dächer vs. die Hypothek auflegen und andererseits spielt man mit der Homophonie, wie im Text (18) Miezhaus vs. Mietshaus und im (20) Krauses Haar vs. krauses Haar.

Wenn man über Kalauer zu sprechen anfängt, wird es klar, dass die Witze, die als flach und schlecht klassifiziert werden, eigentlich von jedem Rezipienten anders interpretiert werden können, es sei denn, man hat es mit konkreten

\footnotetext{
${ }^{4}$ Vgl. dazu mehr: Kreitling H. (2011), Witz, komm raus, Du bist von Kalauern umgeben (=http:// www.welt.de/reise/nah/article13407325/Witz-komm-raus-Du-bist-von-Kalauern-umgeben.html)

${ }^{5}$ Ebd.
} 
Wortspielen zu tun, in denen sprachliche Mittel zu den humoristischen Zwecken absichtlich eingesetzt wurden. Die erwähnten Beispiele zeugen eher davon, dass die Sprache ganz geschickt verwendet wird, wenn ein Text eben ein humoristischer werden sollte.

Anders sieht es in dem Falle aus, wenn das zweite Element der Definition mitberücksichtigt wird, nämlich die Kategorie „schlecht“, „flach“. Dann wird davon ausgegangen, was für Thema, Zielscheibe und Pointe der jeweilige Witz hat. Verstößt der Autor gegen Normen und Regeln, vor allem gegen die sozialen? Wie wird das alles dann wahrgenommen?

Wenn man die sog. Kalauer geschichtlich zu betrachten versucht, fällt es auf, dass sich im Laufe der Zeit die Grenzen verschoben haben, sodass ein Witz von früher heute sogar als nicht mehr lustig, eher als ein neutraler Text verstanden wird. Was vorher als lustig, aber flach galt, kann heute nicht mehr so sein. Alles ist vom Rezipienten und seinem Sinn für Humor abhängig. Der Witz bleibt der alte, nur der Rezipient ist neu, mit seinem eigenen Sinn für Humor, der sich von dem seiner Vorfahren unterscheidet. Auf diese Weise kommt es dazu, dass ein und derselbe Text nach Jahren ganz anders verstanden und interpretiert werden kann. So ist es nämlich mit den Texten von Loriot, die zwar immer noch aktuell bleiben, sich aber gleichzeitig von den von heute sprachlich unterscheiden. Stollmann (2014) bewertet den Humor von Loriot folgendermaßen:

Alles Lachen entsteht durch Kitzeln, und zwar nicht nur durch körperliches Kitzeln. Wenn man z. B. Loriot anschaut, dann ist es so, dass er quasi die nationale Haut der Deutschen kitzelt und an ihr die sensiblen Stellen 6 .

Aus diesen Worten kann man schlussfolgern, dass Loriots Texte immer noch aktuell bleiben.

An dieser Stelle soll man einen der bekanntesten Sketche Loriots aus den 70er Jahren anführen, in dem das Thema Beziehung, das Motiv Feierabend und die Zielscheibe Frau zu nennen sind:

(21) Der Feierabend ${ }^{7}$

Frau: Hermann?

Mann: Ja?

Frau: Was machst du da?

Mann: Nichts.

Frau: Nichts? Wieso nichts?

Mann: Ich mache nichts.

${ }^{6}$ Rainer Stollmann ist Kulturwissenschaftler und Lach-Forscher von der Universität Bremen. Dieses Zitat erscheint in einem Interview, das auf der Internetseite http://www.br.de/fernsehen/ardalpha/sendungen/alpha-forum/rainer-stollmann-sendung-100.html veröffentlicht wurde (Zugriff am 20.08.2015).

${ }^{7}$ Feierabend, Loriot III, Radio Bremen, 16.05.1977. 
Frau: Gar nichts?

Mann: Nein.

Frau: Überhaupt nichts?

Mann: Nein, ich sitze hier.

Frau: Du sitzt da?

Mann: Ja.

Frau: Aber irgendwas machst du doch?

Mann: Nein.

Frau: Denkst du irgendwas?

Mann: Nichts besonderes.

Frau: Es könnte ja nicht schaden, wenn du mal etwas spazieren gingest.

Mann: Nein, nein.

Frau: Ich bringe dir deinen Mantel.

Mann: Nein, danke.

Frau: Aber es ist zu kalt ohne Mantel.

Mann: Ich gehe ja nicht spazieren.

Frau: Aber eben wolltest du doch noch?

Mann: Nein, du wolltest, daß ich spazieren gehe.

Frau: Ich? Mir ist es völlig egal, ob du spazieren gehst.

Mann: Gut.

Frau Ich meine nur, es könnte dir nicht schaden, wenn du mal spazieren gehen würdest.

Mann: Nein, schaden könnte es nicht.

Frau: Also, was willst du denn nun?

Mann: Ich möchte hier sitzen.

Frau: Du kannst einen ja wahnsinnig machen. Erst willst du spazieren gehen, dann wieder nicht. Dann soll ich deinen Mantel holen, dann wieder nicht. Was denn nun? Mann Ich möchte hier sitzen.

Frau: Und jetzt möchtest du plötzlich da sitzen.

Mann: Gar nicht plötzlich. Ich wollte immer nur hier sitzen.

Frau: Sitzen?

Mann: Ich möchte hier sitzen und mich entspannen.

Frau: Wenn du dich wirklich entspannen wolltest, würdest du nicht dauernd auf mich einreden.

Mann: Ich sag' ja nichts mehr.

Frau: Jetzt hättest du doch Zeit, irgendwas zu tun, was dir Spaß macht.

Mann: Ja.

Frau: Liest du was?

Mann: Im Moment nicht.

Frau: Dann lies doch mal was.

Mann: Nachher, nachher vielleicht.

Frau: Hol dir doch die Illustrierten.

Mann: Ich möchte erst noch etwas hier sitzen.

Frau: Soll ich sie dir holen?

Mann: Nein, nein, vielen Dank.

Frau: Will der Herr sich auch noch bedienen lassen, was? Ich renne den ganzen

Tag hin und her. Du könntest wohl einmal aufstehen und dir die Illustrierten holen.

Mann: Ich möchte jetzt nicht lesen.

Frau: Mal möchtest du lesen, mal nicht. 
Mann: Ich möchte einfach hier sitzen.

Frau: Du kannst doch tun, was dir Spaß macht.

Mann: Das tue ich ja.

Frau: Dann quengel doch nicht dauernd so rum. Hermann? Bist du taub ?

Mann: Nein, nein.

Frau: Du tust eben nicht, was dir Spaß macht. Stattdessen sitzt du da.

Mann: Ich sitze hier, weil es mir Spaß macht.

Frau: Sei doch nicht gleich so aggressiv.

Mann: Ich bin doch nicht aggressiv.

Frau: Warum schreist du mich dann so an?

Mann: Ich schreie dich nicht an!

Im erwähnten Text fällt vor allem die Struktur auf, die als Frage-Antwort-Spiel zu verstehen ist. Der ,arme“ Mann wird ständig von seiner Frau angesprochen, obwohl er eigentlich „nur“ seine Ruhe haben möchte. Als Mittel des Humoristischen werden hier vor allem die von der Frau mit viel Biss formulierten Fragen eingesetzt, aus denen hervorgeht, dass sie eigentlich das letzte Wort hat und von ihrem Mann nur Gehorsamkeit erwartet.

Loriot spielt hier eher mit dem situativen Kontext, der mit dem sprachlichen auf eine besondere Art und Weise verstärkt wird. Was den Charakter des Witzes anbelangt, könnte man wagen zu sagen, dass dieser Text für einen Mann gar nicht lustig ist, wobei eine Frau als Rezipientin doch darüber lachen könnte. Selbst daraus resultiert die Annahme, dass ein und derselbe Text auch seine geschlechtsspezifischen Merkmale haben kann, die auch Einfluss auf die Reaktion der Zuhörer bzw. Zuschauer haben können. Ob das aber ein Kalauer ist, könnte man natürlich diskutieren. Als flacher Witz soll ein Text im ersten Moment doch gar nicht so wirken, als ob er ein Witz wäre, man macht sich dann nur Gedanken darüber, wozu solche Witze überhaupt geschrieben werden. Der Text von Loriot stammt aus dem Jahre 1977. Zum Vergleich kann man an dieser Stelle ein paar Szenen aus „Was guckst du?" ${ }^{\circ}$ heranziehen, die seit Ende der 90er Jahre des 20. Jahrhunderts geschrieben wurden, also über 20 Jahre nach Loriot. Die Hauptfigur ist Francesco, der sich im Restaurant mit vielen Frauen trifft, sich jedes Mal aber völlig daneben benimmt. In diesem Fall spielt neben der eingesetzten Sprache auch die Körpersprache $^{9}$ eine Rolle, die für jeweilige Figuren charakteristisch ist. Mit ihrer Hilfe wird die Wirkung der sprachlichen Mittel nur zusätzlich verstärkt.

${ }^{8}$ Das Programm „Was guckst du?““ ist ein multikulturelles Comedy-Programm, das von einem türkisch-arabischen, in Deutschland geborenen Komiker - Kaya Yanar - ins deutsche Fernsehen gebracht und dort moderiert wurde. Für seine Sendung bekam er zahlreiche Preise, u.a. den Deutschen Fernsehpreis 2001 in der Kategorie „Beste Comedy“.

${ }^{9}$ Man soll an dieser Stelle auf Matschnig hinweisen, die sich in ihren Artikeln mit den Fragen der Körpersprache auseinandersetzt. Selbst die Titel ihrer Beiträge werten schon die Körpersprache als Mittel der Kommunikation: „Ein Hauch von Nichts“ entscheidet (2007a), Gesten sagen mehr als Worte. Die Körpersprache gibt den Ton an. (2007b), Zuerst spricht der Körper. (2011a), Die 
(22) Francesco im Restaurant 1

Francesco: O, Bella, ich habe noch nie so eine Frau gesehen... Er dreht seinen Kopf zur Seite

Frau: Ja?

Francesco: ... die so schnell essen kann Er dreht seinen Kopf zur Seite... und Er wiederholt die Kopfbewegung so viel Er sieht sich um, um zu prüfen, ob jemand sie beobachtet oder ihnen vielleicht zuhört... und so teuer!

(23) Francesco im Restaurant 2

Francesco: O, Bella, kannst du mir sagen, was ist das Parfüm, das du benutzt? Er zieht die Augenbrauen hoch, was von der Frau als Interesse an ihr und ihrem Parfüm verstanden wird.

Frau: Wieso? Gefällt es dir? Sie sieht so glücklich aus.

Francesco: No, no, no! Es überdeckt deinen Mundgeruch! Die Frau ist empört, steht auf und geht weg.

(24) Francesco im Restaurant 3

Francesco: Ich suche eine Frau, die nicht nur kochen kann...

Frau: Ja? Die Frau sieht so aus, als ob sie in diesem Moment auf den Heiratsantrag von Francesco warten würde.

Francesco: ...sondern auch backen! Die Frau ist äußerst enttäuscht, steht auf und geht weg.

Aus der Analyse dieser erwähnten kurzen Texte geht hervor, dass die Männerfigur nicht durch die Blumen redet, sondern alles direkt formuliert. Die Frau wird als solche dargestellt, die ständig von dem Mann überrascht werden möchte, aber dabei auf solche Art und Weise, dass alle ihre Wünsche in Erfüllung gehen. Dem Mann ist es eigentlich egal, was sie denkt, er sagt, was er zu sagen hat. Eine neutrale Sprache in Verbindung mit dem derben Verhalten und ehrlich gesagt bösen Absichten - das alles bildet eine Mischung, die den Rezipienten im Endeffekt zum Lachen bringt. Ist das aber ein Zeichen dafür, dass das ein guter Witz ist? Oder beurteilen wir diese Szenen so, weil uns dieser Mann sympathisch zu sein scheint und weil uns die Frau eher Leid tut, weil sie sich bei ihm nur anschmeicheln will?

Die Frage kann nicht ein für alle Male beantwortet werden, weil das Ganze auf die rein subjektive Wahrnehmung dieses witzigen Textes zurückgeht und darauf, was für den Rezipienten ein Kalauer ist. Versteht er ihn als einen Witz, wo man mit Wortspielen geschickt umgeht oder vielleicht doch als einen flachen, schlechten Witz? Erst die Antwort auf eine so formulierte Frage könnte den Ausgangspunkt der ganzen Diskussion über die Frauen-Männer-Witze bilden, weil man sich dann von Anfang an nur nach einem eindeutigen Kriterium richten würde.

Körpersprache der Macher. (2012):, Der Eindruck zählt. (2011b). Der Körpersprache wird eine sehr wichtige Rolle zugeschrieben, vor allem wegen ihrer sofortigen Wirkung bei dem Gegenüber. 


\section{Fazit und Prognosen}

Aus der für die Zwecke dieses Beitrags durchgeführten Analyse geht eindeutig hervor, dass die Frauen-Männer-Witze sogar nach den beiden Kriterien untersucht und beurteilt werden können, weil sie einerseits sehr oft so eine Struktur haben, dass man es dort mit den Wortspielen zu tun hat und andererseits doch dem Inhalt nach eher der Gruppe der schlechten Witze zugeordnet werden können, was wiederum auf ihren Charakter zurückgeht, der mit der Art und Weise der Darstellung der Figuren, mit den im Hintergrund stehenden, nicht selten sogar dummen Gedanken verbunden ist. Es muss jedoch dabei betont werden, dass mit einem Kalauer in erster Linie ein schlechter, flacher, nicht geistreicher Witz assoziiert wird.

Die Klassifikation der Witze nach rein sprachlichen Kriterien ist zeitunabhängig. Anders ist es im Falle der Analyse der Struktur der Witze und der Wahrnehmung des Grundgedankens der humoristischen Texte. Die Zeit vergeht und mit ihr ändert sich auch der Zuschauer bzw. Zuhörer, was auf die Veränderungen im gesellschaftlichen Leben zurückzuführen ist. Die Texte bleiben als Erbgut erhalten, die Nachkommen betrachten sie aber auf solche Art und Weise und nach solchen Kriterien, die für ihre Zeiten charakteristisch sind und nicht danach, wie es vor Jahren noch war, als die Texte entstanden sind.

Da die elektronischen Medien heutzutage als die größte zugängliche Quelle der Witze gesehen werden, steht es außer Zweifel, dass alle heute angebotenen humoristischen Texte weiter leben werden, ohne dass sie in einer gedruckten Form erscheinen. Wegen der Anonymität im Netz werden jeden Tag neue Witze geschrieben und eigentlich lässt sich nicht eindeutig sagen, wie die Autoren bewertet werden sollen, weil diese Namen leider meistens unbekannt bleiben.

\section{LITERATURVERZEICHNIS}

Attardo S. (1994), Linguistic Theories of Humour, de Gruyter, Berlin/New York.

Attardo S., Raskin V. (1991), Script Theory Revis(it)ed: Joke Similarity ans Joke Representation Model. In: Humor, Bd. 3: 3/4, S. 293-347.

Bergson H. (1900/1977), Śmiech. Esej o komizmie, polnische Übersetzung von S. Cichowicz, Kraków.

Bremmer J., Rodenburg H. (Hrsg.) (1999), Kulturgeschichte des Humors - Von der Antike bis heute, Darmstadt.

Buttler D. (1968/2001), Polski dowcip językowy, Warszawa.

Dimova A. (2000), Humor und Witz als Übersetzungsproblem. In: Hoffmann T., Lercher M-Ch., Middeke A., Tittel K.(Hrsg.), Humor. Grenzüberschreitende Spielarten eines kulturellen Phänomens, Göttingen, S. 7-20.

Freud S. (1905/1985), Der Witz und seine Beziehung zum Unbewußten, Frankfurt/ M.

Freud S. (1927/1982), Der Humor. Studienausgabe, Bd. 4, Frankfurt/M. 
Kotthoff H. (1986), Scherzen und Lachen in Gesprächen von Frauen und Männern. In: Der Deutschunterricht, Nr. 3, S. 16-29.

Kotthoff H. (1988), Das Gelächter der Geschlechter. Humor und Macht in Gesprächen von Frauen und Männern, Frankfurt/M.

Kotthoff H. (1992), Von gackernden Hühnern und röhrenden Hirschen. Konversationelles Scherzen zwischen Männern und Frauen. In: Vogel Th. (Hrsg.), Vom Lachen, Tübingen, S. 192-209.

Kotthoff H.(1996), Scherzkommunikation. Beiträge aus der empirischen Gesprächsforschung, Opladen.

Kotthoff H. (1998a), Witzige Darbietungen auf eigene Kosten. Über Komplexitäten weiblicher Imagepolitik in der Scherzkommunikation. In: Zeitschrift für germanistische Linguistik, Bd. 139/140, S. 253-279.

Kotthoff H. (1998b), Spaß Verstehen. Zur Pragmatik von konversationellem Humor, Niemeyer Verlag, Tübingen.

Kotthoff H. (2000), Konversationelle Parodie. Über komische Intertextualität in der Alltagskommunikation. In: Germanistische Linguistik, Bd. 153, S. 159-186.

Kotthoff H. (2002a), Ironie in Privatgesprächen und Fernsehdiskussionen. Zum Zusammenhang von Konversation, Kognition und Ethnographie. In: Keim I., Schütte W.(Hrsg.), Soziale Welten und kommunikative Stile. Festschrift für Werner Kallmeyer, Tübingen, S. 445-473.

Kotthoff H. (2002b), Über die Zähmung des weiblichen Gelächters. In: Chauré E., Gutknecht O., Schmidt C. (Hrsg.), Zur Konstruktion von Geschlecht in Sprache, Gesellschaft und Kultur. Gedächtnisschrift für Gisela Schoenthal, Freiburg, S. 10-3113.

Kotthoff H. (2003), Lachkulturen heute: Humor in Gesprächen. In: Roters G., Klingler W. (Hrsg.), Forum Medienrezeption, Baden-Baden, S. 45-75.

Kotthoff H. (2004), Geschlechterverhältnisse in der Scherzkommunikation: Althergebrachtes und neue Trends in der Alltags- und Fernsehkomik. In: Epp H. (Hrsg.), Gender studies - Interdisziplinäre Ansichten 1, Freiburg, S. 15-53.

Kreitling H. (2011), Witz, komm raus, Du bist von Kalauern umgeben. (= http://www.welt.de/reise/ nah/article13407325/Witz-komm-raus-Du-bist-von-Kalauern-umgeben.html) Zugriff am 24.07.2013.

Matschnig M. (2007a), „Ein Hauch von Nichts “ entscheidet. In: Weser - Kurier 5/2007 (= http:// matschnig.com/de/images/pdf/WeserKurier.pdf, Zugriff am 24.07.2013).

Matschnig M.(2007b), Gesten sagen mehr als Worte. Die Körpersprache gibt den Ton an, In: telis magazin 2007, S.142-144( =http://matschnig.com/files/TELIS.pdf, Zugriff am 24.07.2013).

Matschnig M.(2011a), Zuerst spricht der Körper. In: Kärtner Wirtschaft 10/2011 (=http://matschnig.com/de/images/MX-2600N_20111007_100213.pdf, Zugriff am 24.07.2013).

Matschnig M. (2012), Die Körpersprache der Macher. In: Ruhrzeitung 09/2012(= http://matschnig. com/home/wp-content/uploads/2012/02/Koerpersprache.pdf, Zugriff am 24.07.2013).

Matschnig M. (2011b), Der Eindruck zählt: Was uns die Körpersprache sagt. In: BadischeZeitung vom 18.01.2011 (= http://www.badische-zeitung.de/liebe-familie/der-eindruck-zaehlt- wasuns-die-koerpersprache-sagt--40096498.html, Zugriff am 18.01.2011).

Raskin V. (1985), Semantic Mechanism of Humour, Dordrecht.

Sikorska-Bujnowicz K. (2013), Sprachliche Mittel des Humoristischen im Deutschen und imPolnischen, Łódź.

Stollmann R. (2015), Ausdruck des Verstands. Kein Witz: Wer schlauer ist, lacht länger (= http:// www.focus.de/wissen/experten/stollmann/auf-den-punkt-gebracht-was-genau-ist-ein-witz id_4519899.html, Zugriff am 24.07.2013).

Titze M.(2009a), Wie komisch ist der Humor? Über Erheiterung, Lachen, Schadenfreude, Inkongruenz und Ironie. In: TPS - Theorie und Praxis der Sozialpädagogik, Bd. 6, S. 20-25 (von http:// www.michael-titze.de/, Zugriff am 10.05.2012). 
Titze M.(2009b), Die disziplinarische Funktion der Schadenfreude - oder: Die Ambivalenz des Lachens. In: Wahl P., Sasse H., Lehmkuhl U. (Hrsg.), Freude - Jenseits von Ach und Weh? (= Beiträge zur Individualpsychologie, Bd. 37), Göttingen, S. 11-39 (gekürzt) (von http://www. michael-titze.de/, Zugriff am 10.05.2012).

\section{INTERNETQUELLEN}

www.br.de/fernsehen/ard-alpha/sendungen/alpha-forum/rainer-stollmann-sendung-100.html (=Rainer Stollmann im Gespräch mit Jutta Henkel) Zugriff am 20.08.2015.

www.gutenberg.spiegel.de, Zugriff am 24.07.2013.

www.programmwechsel.de, Zugriff am 24.07.2013.

www.spitzenwitze.de, Zugriff am 24.07.2013.

www.teilenswert.de, Zugriff am 24.07.2013.

Katarzyna Sikorska-Bujnowicz

\section{WOMEN-MEN-JOKE THEN AND NOW. SOME COMMENTS ON THE GERMAN PUN}

(Summary)

In this article an attempt is made to discuss the German joke regarding its assignment to the class of bad jokes in German. The question of what is a pun and what determines that a humorous text can be classified as bad or good is also important and very interesting. No less important are questions about the possibility of breaking all rules and norms, both of linguistic and social natures. These considerations are documented by examples of German jokes; on the one hand Loriot's texts, texts from "Was guckst du?" and on the other from websites. The question of whether an analysed joke is good or bad still remains open despite all possible classification attempts, because its interpretation is subjective, to be assessed for what it is by the recipient from the outset.

Key words: humour studies, bad jokes in German, boundaries of good taste in a joke, joke and rule, German sense of humour. 